\title{
Insertion sequence-excision enhancer removes transposable elements from bacterial genomes and induces various genomic deletions
}

\author{
Masahiro Kusumoto', Tadasuke Ooka², Yoshiaki Nishiya ${ }^{3}$, Yoshitoshi Ogura'2,4, Takashi Saito ${ }^{5}$, \\ Yasuhiko Sekine ${ }^{5}$, Taketoshi Iwata', Masato Akiba' \& Tetsuya Hayashi²,4
}

Insertion sequences (ISs) are the simplest transposable elements and are widely distributed in bacteria. It has long been thought that IS excision rarely occurs in bacterial cells because most bacteria exhibit no end-joining activity to regenerate donor DNA after IS excision. Recently, however, we found that excision of IS629, an IS3 family member, occurs frequently in Escherichia coli O157. In this paper, we describe a protein IS-excision enhancer (IEE) that promotes IS629 excision from the $\mathrm{O} 157$ genome in an IS transposase-dependent manner. Various types of genomic deletions are also generated on IEE-mediated IS excision, and IEE promotes the excision of other IS 3 family members and ISs from several other IS families. These data and the phylogeny of IEE homologues found in a broad range of bacteria suggest that IEE proteins have coevolved with IS elements and have pivotal roles in bacterial genome evolution by inducing IS removal and genomic deletion.

\footnotetext{
1 Safety Research Team, National Institute of Animal Health, 3-1-5 Kannondai, Tsukuba, Ibaraki 305-0856, Japan. ${ }^{2}$ Division of Microbiology, Department of Infectious Diseases, Faculty of Medicine, University of Miyazaki, Miyazaki 889-1692, Japan. ${ }^{3}$ Tsuruga Institute of Biotechnology, Toyobo Co., Ltd., Fukui 914-0047, Japan. ${ }^{4}$ Division of Bioenvironmental Science, Frontier Science Research Center, University of Miyazaki, Miyazaki 889-1692, Japan. ${ }^{5}$ Department of Life Science, College of Science, Rikkyo University, Tokyo 171-8501, Japan. Correspondence and requests for materials should be addressed to M.K. (email: kusu555@affrc.go.jp).
} 
$\mathrm{T}$ ransposable elements (TEs), or transposons (Tns), are widely distributed in eukaryotic and prokaryotic genomes. They are considered selfish (or parasitic) genetic elements, but they also have important roles in genome evolution ${ }^{1}$. Insertion sequence (IS) elements, which are the simplest TEs, are 700-2,500 bp in size and encode only the transposase (TPase) catalysing their own transposition $^{2}$. The transposition and proliferation of IS elements induce not only insertional gene inactivation and modification of gene expression $^{1}$ but also a variety of genomic rearrangements, such as deletions, inversions and duplications $s^{3,4}$.

In bacteria, several thousand types of IS elements have thus far been identified from various species and strains $s^{5}$. These elements are classified into $\sim 20$ families on the basis of the sequences of their TPases and terminal inverted repeats (TIRs), in addition to several other features ${ }^{2}$. The mechanism of transposition differs between IS families, but IS elements transpose and either leave the original copy in the donor DNA (termed copy-and-paste, or replicative, transposition) or eradicate it from the donor DNA (cut-and-paste, or non-replicative, transposition) ${ }^{6}$.

The mechanisms of transposition have been intensively studied for representative IS elements of several families, and one of the best studied is the IS3 family. The process of transposition in this large IS family is initiated by the formation of a 'figure-eight intermediate, which is followed by the generation of an excised circular DNA molecule that is randomly inserted into the target $\mathrm{DNA}^{2}$. Although the entire process is not yet fully understood at the molecular level, it has been demonstrated for IS911, a representative family member, that a replicative pathway is used to generate a circular DNA molecule from the figure-eight intermediate. Thus, IS911 transposes by the copy-and-paste mechanism 7 . If the copy-and-paste mechanism is the major pathway for the transposition of the IS3 family, the excision of IS3 family members occurs very rarely in the normal transposition process.

In general, researchers believe that the excision of IS elements is a rare genetic event in bacteria ${ }^{8}$, because end-joining systems, which are required to reseal the donor DNA for its survival after IS excision, have been identified in relatively few bacterial species? Thus, very little attention has been paid to the genetic events that occur to donor DNA on IS excision. Tn excision in bacteria has been described in several reports, but such excision was observed to be independent of TPase activity. Instead, it is dependent on host factors required for replication slippage and repair functions ${ }^{10,11}$. In practise, these excision events have been observed at a low frequency. However, we recently obtained several lines of evidence to show that excision of IS629, a member of the IS3 family, occurs frequently in the enterohaemorrhagic Escherichia coli (EHEC) strain O157.

EHEC O157 produces highly potent cytotoxins (Shiga toxins Stx1 and/or Stx2) and causes diarrhoea, haemorrhagic colitis and haemolytic uremic syndrome, thus being regarded as one of the most serious worldwide food-borne infections ${ }^{12}$. The 0157 strain that has been sequenced (RIMD0509952, referred to as O157 Sakai) contains 25 types of IS elements (116 copies in total) ${ }^{13}$. Among these elements, the most abundant is IS629 (also called IS1203v): 23 copies are present on the bacterial chromosome and on the pO157 virulence plasmid. Of these copies, 17 (74\%) are apparently intact, whereas only 22 copies (24\%) are intact among other types of IS elements, suggesting that active copies of IS629 are selectively maintained in EHEC O157. In fact, our comparative genomic analysis of EHEC O157 clinical isolates revealed that many small-sized structural polymorphisms associated with gene inactivation and/or deletion have been generated by IS629 in EHEC O157 strains ${ }^{14}$. Several genomic loci in which IS629 has been deleted by simple excision were also identified.

In another series of studies, we observed that an stx2 gene that was inactivated by IS629 insertion, which was found in some O157 clinical isolates ${ }^{15,16}$, was reactivated by precise IS629 excision in the E. coli laboratory strain K-12 (but at a very low frequency) ${ }^{17}$. However, using a reporter plasmid-based assay, we found that precise IS629 excision occurs much more frequently in O157 Sakai than in K-12, and several other EHEC strains also exhibit a high frequency of IS629 excision ${ }^{18}$. These results suggest that some E. coli strains, including O157, may contain a previously unknown system to promote IS excision by regenerating the donor DNA lacking the IS copy. The clear difference between O157 Sakai and K-12 in excision frequency further suggests that this system may be encoded by one or more of the $\sim 1,600$ O157 Sakai genes that are not present in K-12 (ref. 19).

Here, we describe an O157 Sakai gene that promotes the excision of IS629 and of several other ISs in a TPase-dependent manner and generates various types of genomic deletions on IS excision, thus having a pivotal role in bacterial genome evolution. We further identify many homologues of this gene, which appear to have been spread by horizontal gene transfer and to have been coevolved with IS elements, in a wide range of bacteria.

\section{Results}

Identification of a protein that enhances IS629 excision. To identify a genetic determinant(s) that is responsible for a high frequency of IS629 excision in O157 and other EHEC strains, we first analysed, using a reporter plasmid (Fig. 1a), the frequencies of precise IS629 excision in K-12, O157 Sakai and 13 other EHEC strains of the O157, O26, O111 or O103 serotype, the whole gene repertoires of which were previously analysed by comparative genomic hybridization using an O157 oligo DNA microarray ${ }^{20}$. Among the 15 strains tested, 11 EHEC strains (including O157 Sakai) exhibited a high excision frequency, whereas 4 strains (O157 \#3, O26 \#1, O103 \#6 and K12) showed no or an extremely low excision frequency (Fig. 1b). By reinspecting the comparative genomic hybridization data for these 15 strains, we found that ECs1305, which encodes a hypothetical protein, was the only gene that was present in the high frequency group but not in the low frequency group.

We detected no precise IS629 excision in an ECs1305-deletion mutant of O157 Sakai (strain GMSS401 in Fig. 1c), and the excision frequency of the mutant returned to the parental level by reintroducing ECs1305 (GMSS401(pIEE1)). Furthermore, K-12 derivatives containing ECs1305 (chromosomally inserted or plasmid encoded) showed a high IS629 excision rate comparable to or higher than that in O157 Sakai (GMEC101 and K-12(pIEE1) in Fig. 1c). These results indicate that ECs1305 enhances precise IS629 excision in the genetic backgrounds of $\mathrm{O} 157$ and $\mathrm{K}-12$. We also confirmed that the presence of the antibiotics used as selecting agents resulted in no statistically significant effect on the IS629 excision frequencies.

To determine whether the ECs1305-mediated enhancement of IS629 excision is a process dependent on TPase activity, we constructed three reporter plasmids encoding mutant IS629 TPases. In these mutants, one of the three acidic amino-acid residues constituting the DDE motif, which represents the active centre of the IS TPase $^{2}$, was substituted with alanine (D244A, D303A and E339A). K-12 derivatives carrying a plasmid-encoded ECs1305 and the plasmids expressing these mutant TPases exhibited no IS629 excision (Supplementary Fig. S1). This result indicates that the ECs1305-mediated enhancement of IS629 excision requires an active IS629 TPase.

To determine whether other host factors are also involved in the excision of IS629 by ECs1305, we constructed a series of O157 Sakai mutants in which genes encoding host factors that have been demonstrated or suggested to be involved in the regulation of Tn transposition or TPase-independent IS deletion were deleted (Supplementary Fig. S2a). These gene products may be involved in the transposition or deletion of IS elements at various levels, such as the expression of TPase genes, transpososome assembly, replication slippage or DNA repair (see refs 2, 21 for reviews). Because we were unable to delete 
a

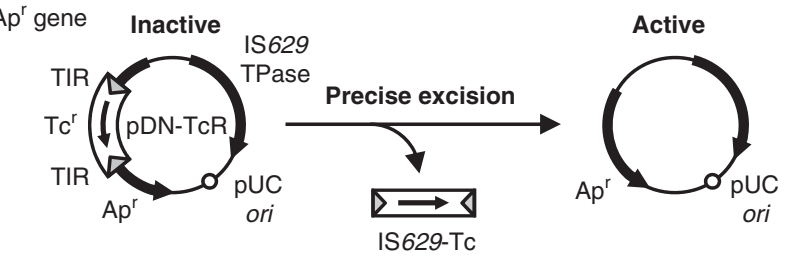

b

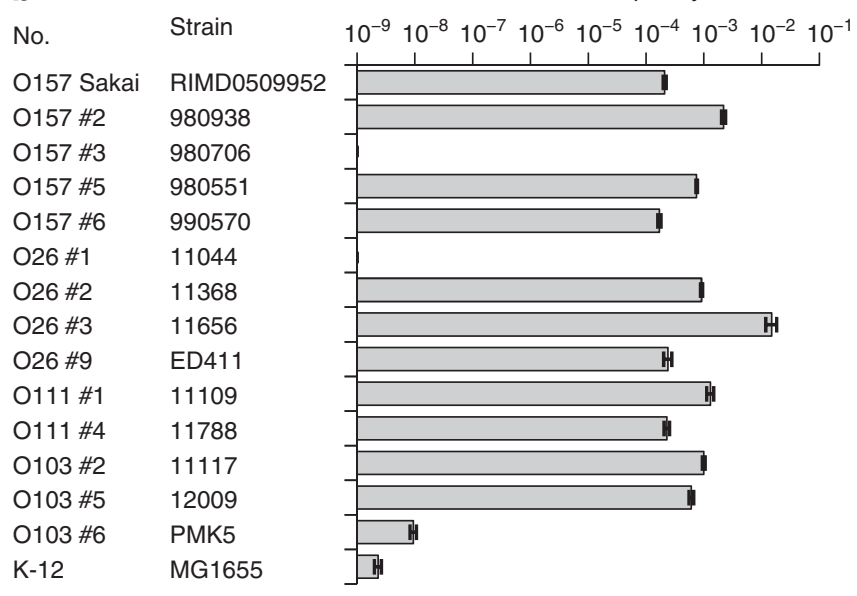

C

Strain

O157 Sakai

GMSS401

GMSS401(pIEE1)

GMEC101

$\mathrm{K}-12$

$\mathrm{K}-12($ plEE1) iee

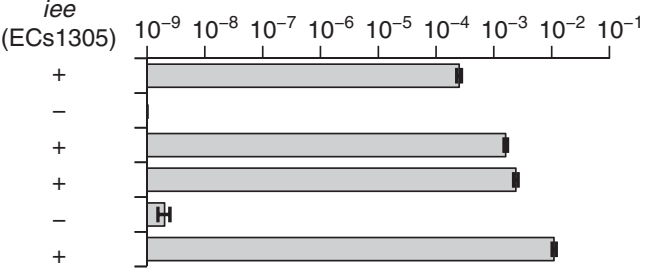

Figure 1 | IS629 excision frequencies in various $E$. coli strains. (a) Schematic representation of the reporter plasmid assay for analysing the frequency of the precise excision of IS629. (b) Frequencies of precise IS629 excision in K-12, 0157 Sakai and another 13 EHEC strains of O157, O26, $\mathrm{O} 111$ and $\mathrm{O} 103$ serotypes. The excision frequency was determined using the reporter plasmid assay shown in panel (a) and calculated by dividing the number of ampicillin-resistant $\left(A p^{r}\right)$ colonies by that of tetracyclineresistant $\left(T c^{r}\right)$ colonies (mean \pm s.d., $n=3$ ). Four strains $(0157 \# 3,026 \# 1$, 0103 \#6 and K-12) exhibited radically lower excision frequencies $\left(<10^{-7}\right)$ compared with the other 11 strains $\left(>10^{-4}\right.$ ), which included 0157 Sakai. No Apr colonies were obtained for 0157 \#3 or 026 \#1. (c) The effects of ECs1305 (the iee gene) on IS629 excision frequency in O157 Sakai, K-12 and their derivatives. ECs1305 is encoded by a large integrative element called SpLE1 in the genome of O157 Sakai. GMSS401 is an iee-deletion mutant of 0157 Sakai. GMEC101 is a K-12 derivative in which the iee gene was inserted into an IS3 copy on the chromosome. GMSS401(pIEE1) and $\mathrm{K}-12$ (pIEE1) contain a plasmid carrying the iee gene. The excision frequency was determined as in panel (b). Plus and minus symbols indicate the presence and absence of the iee gene in each strain, respectively. No Apr colonies were obtained for GMSS401.

the hns, dam and recB genes in O157 Sakai, we constructed deletion mutants for these three genes from GMEC101, a K-12 derivative containing a chromosomally inserted ECs1305 (Supplementary Fig. S2b). Many of these deleted genes affected ECs1305-mediated IS629 excision in various ways (Supplementary Fig. S2). However, the increase or decrease of the IS629 excision frequency induced by the deletion of these genes was only marginal (at most, $\sim 50$-fold) compared with the effect of ECs1305 deletion (>106-fold decrease). Thus, the roles of these host factors in the observed IS629 excision are minor or indirect. For example, the excision frequency slightly increased in the absence of host factors, which would be expected to affect the stability of ECs1305 and/or IS TPase (the ClpXP protease) or their gene expression (the H-NS protein). The frequency of IS629 excision decreased in a mutant lacking HU, which is a histone-like protein that may assist in transpososome assembly or in other processes requiring higher-order DNA structures. Some reduction was also observed for the excision frequency in mutants lacking RecA, $\mathrm{B}$ or $\mathrm{C}$ proteins, suggesting that DNA repair processes mediated by these proteins contribute only slightly to the observed IS629 excision.

Taken together, our results indicate that ECs1305 has an essential role in high-frequency IS629 excision in an IS TPase-dependent manner, although it is possible that some unknown host factor(s) is(are) also required for the process. Therefore, we designated ECs1305 as an IS-excision enhancer (IEE).

IEE-promoted IS excision induces various genomic deletions. We next examined whether IEE actually promotes the excision of IS629 copies in the O157 Sakai genome using a multiplex PCR-based method (the IS-printing method) that we recently developed for O157 strain typing ${ }^{22}$. Using this method, most of the IS629 copies in the $\mathrm{O} 157$ Sakai genome can be detected by production of a ladder band pattern using two sets of multiplex PCR primers (the first and second sets in Fig. 2a-d), each comprising an IS629 inside primer and multiple primers targeted to the flanking regions for each IS629 element. Two IS629 copies that are not included in the IS-printing system were examined using a similar system (the third set in Fig. 2a-d). Thus, we were able to detect structural alterations occurring at any of the IS629 insertion sites in the O157 Sakai genome (that is, deletion of IS629 and/or flanking regions) by inspecting the ladder patterns (Fig. 2a-d). After O157 Sakai cells possessing both the IS629 TPase- and the IEE-expressing plasmids were cultivated for $24 \mathrm{~h}$ in Luria-Bertani (LB) broth containing L-arabinose to induce IEE expression, 32 single colonies (referred to as Ex06001-Ex06032) were randomly selected and analysed by the IS-printing method. Alterations of the ladder pattern were detected in 29 clones (91\%) in which 1-7 bands disappeared (Fig. 2a). Among the 30 bands, all but 5 that were derived from truncated IS629 copies (IS629-4, -10, -p1, - $\mathrm{pC}$ and $-\mathrm{pD}$ ) were found to be missing in one or more clones (Fig. 2e). No such alteration in the ladder pattern occurred in $\mathrm{O} 157$ Sakai cells containing either the TPase- or the IEE-expressing plasmid alone (Fig. 2b-d). This indicates that IEE, TPase and an IS629 sequence with a complete TIR recognized by TPase in transposition $^{2}$ are required for IEE-induced structural alterations. Notably, none of the O157 Sakai genomic regions containing 19 other types of IS elements (not IS629) with complete TIRs underwent structural changes among the 32 clones (Supplementary Fig. S3).

We further analysed the structures of all IS629 insertion sites that underwent structural alterations (78 sites in 29 clones, Supplementary Fig. S4) and found that various types of genomic deletions were generated by IEE and IS629 TPase. As summarized in Fig. 3, these genomic deletions were categorized into four types: (I) deletion of IS629 with an adjacent short sequence of $1-7$ bp (mainly 3 or 4 bp); (II) deletion of IS629 with an adjacent long sequence; (III) partial deletion of IS629; and (IV) deletion of a long sequence adjacent to IS629. In types I, II and III, additional deletion of a 3-5 bp sequence (mainly $3 \mathrm{bp}$ ) located adjacent to the other end of IS629 was also sometimes observed (types Ib, IIb and IIIb in Fig. 3).

Among the four types of deletions, the most frequently generated was type Ia (51.3\%), which corresponds to precise or simple excision. In the transposition process of IS 3 family members, TPases first cleave one DNA strand at the $3^{\prime}$ end of the IS element ${ }^{7,23}$ (Fig. 4a), and the liberated $3^{\prime}$ end is transferred to the same DNA strand at a position 3 or $4 \mathrm{bp}$ away from the $5^{\prime}$ end of the IS element to generate a figure-eight intermediate (Fig. 4b,c). In the final step, DNA replication from the figure-eight junction generates a 

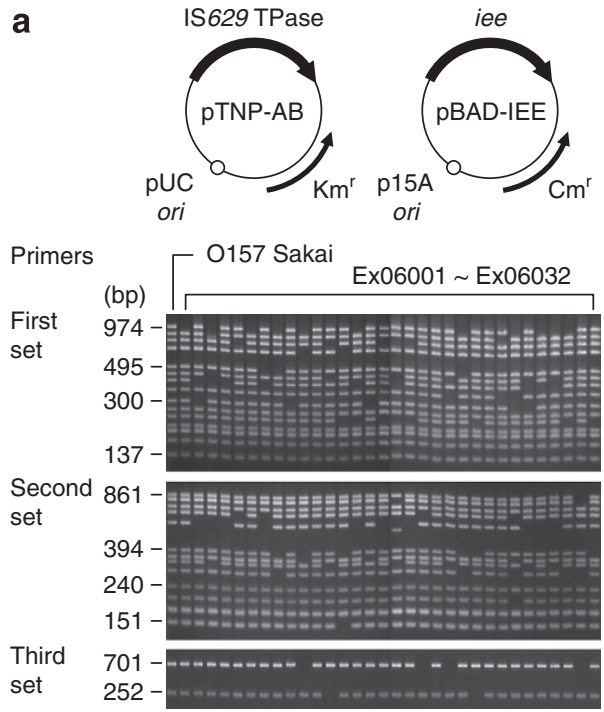

C
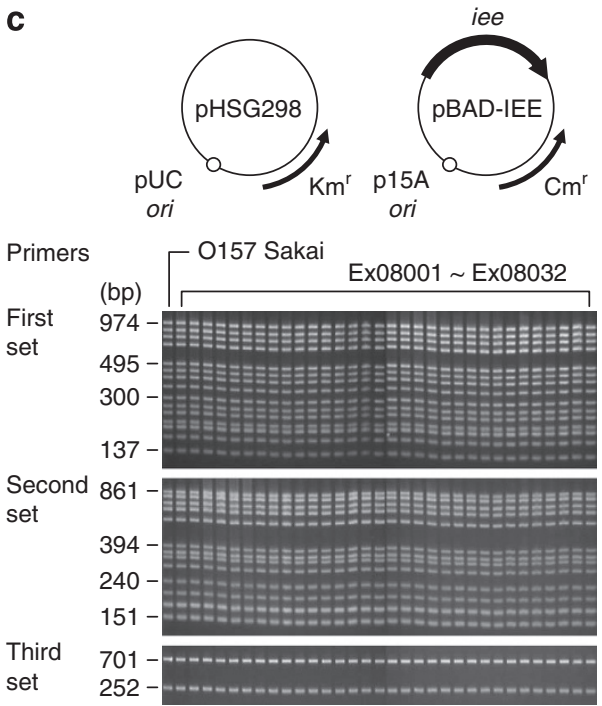
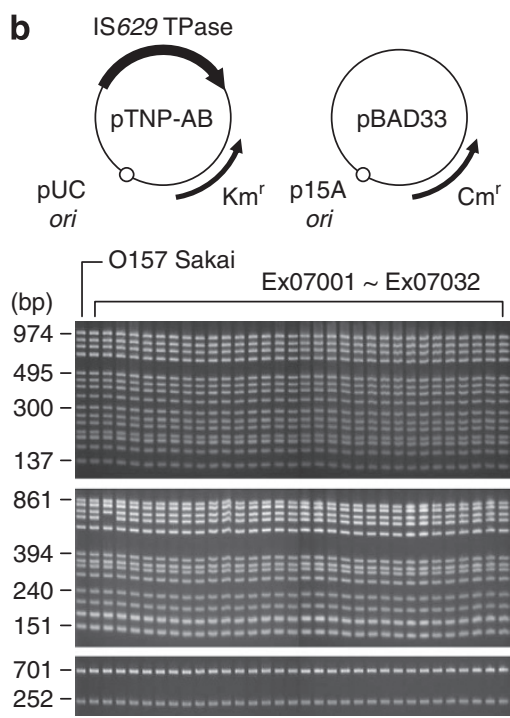

d
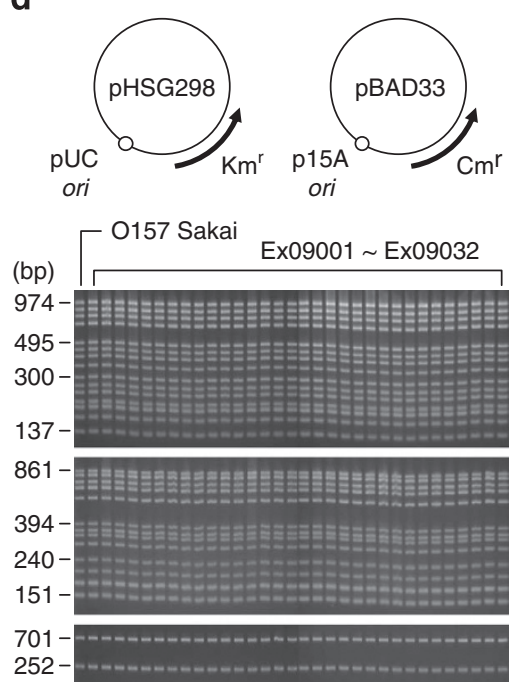

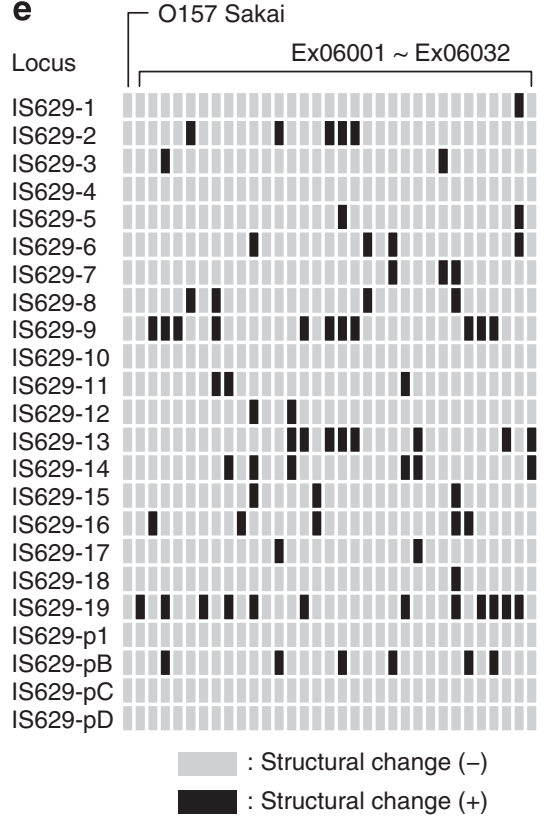

Figure 2 | IS629 TPase- and IEE-induced structural alterations of IS629 insertion sites in the 0157 Sakai genome. Results of IS-printing analyses of the four sets of 0157 Sakai derivatives (32 clones each) that were obtained after overexpression of IS629 TPase and IEE together (a), IS629 TPase alone (b), IEE alone (c) or neither IS629 TPase nor IEE (d) are shown. Structures of the IS629 TPase expression plasmid (pTNP-AB), the IEE expression plasmid (pBAD-IEE) and their control plasmids that were used for overexpression are also shown. Ex06001-Ex06032, Ex07001-Ex07032, Ex08001-Ex08032 and Ex09001-Ex09032 indicate the clone numbers in each set of the 0157 Sakai derivatives. IS-printing analysis was performed using three sets of multiplex PCR primers (the first to third sets). All 23 IS629 insertion sites in the O157 Sakai genome were examined by multiplex PCR followed by agarose gel electrophoresis. The presence or absence of structural alterations at each IS629 insertion site was determined according to the ladder band patterns observed for each clone. (e) The results of IS-printing analysis of clones Ex06001-Ex06032 shown in panel (a) are summarized. Grey and black rectangles indicate IS629 insertion sites without and with structural changes, respectively.

circular transposition intermediate and regenerates the donor DNA containing the original IS copy ${ }^{2,24}$. IEE probably functions in this last step to guide the process towards deleting the IS629 copy from the donor DNA (Fig. 4d). Although the molecular mechanism of this phenomenon has yet to be clarified, second-strand cleavage and resealing of donor DNA seem to be required for IS629 deletion. In several cases, deletion of $1,5,6$ or $7 \mathrm{bp}$ of the adjacent sequence was also observed. These atypical deletions may have been generated by imprecise strand transfer.

The generation of other types of deletion was an unexpected finding. All these variant types were, however, formed only when IEE and the IS629 TPase were coexpressed (Fig. 2), indicating that they were generated in a process coupled with IS excision mediated by IEE and the IS629 TPase. Indeed, none of these variant deletion types were detected in the reporter plasmid assay (Fig. 1a), in which only precise excision was detectable. Notably, all of these variant deletions were observed in naturally occurring $\mathrm{O} 157$ strains ${ }^{14}$. The mechanism(s) to generate these types of deletion is(are) unknown at present. It is also unknown why type IV deletions were generated at a relatively high frequency $(24.4 \%)$. It may be possible to assume that they were generated by aberrant strand transfer (Fig. 4e), but this requires future examination.

It is also important to note that IEE mainly induced the removal of IS629 from the genome (75.6\% of the structural changes). The result of Southern blot analysis of the 32 clones (Ex06001Ex06032) using an IS629-specific probe indicated that the total copy number of IS629 decreased in most clones (Fig. 5). Several new signals that were absent from the parent strain were detected in several clones, but many of these were probably generated by type III or IV deletions. 


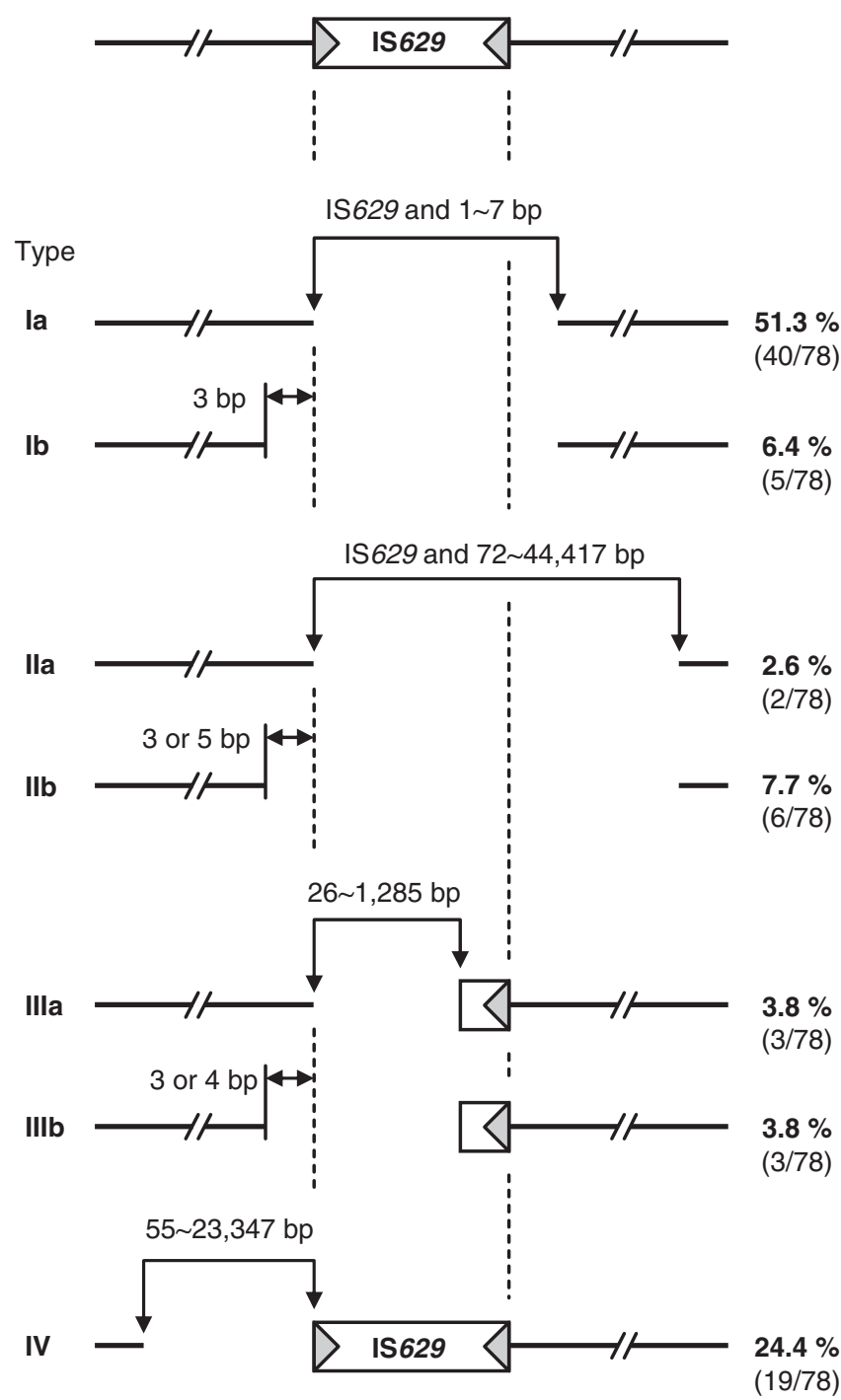

Figure 3 | Various genomic deletions generated by IEE in the presence of IS629 TPase. Structural changes of IS629 insertion sites in the O157 Sakai genome that were induced by IEE in the presence of IS629 TPase were classified into four types. In types I and II, the entire IS629 sequence was deleted, together with short (1-7 bp, mainly 3 or 4 bp) and long (72-44,417 bp) adjacent sequences, respectively. In type III, portions (26-1,285 bp) of the 1,310-bp IS629 sequence were deleted. Deletion occurred at one IS end in types Ia, Ila and IIIa, and additional deletions of adjacent sequences occurred at the other IS end in types Ib, IIb and IIIb. In type IV, only the genomic segments adjacent to IS629 (55-23,347 bp) were deleted without deletion of the IS element. Grey triangles indicate TIRs of IS629. The detection frequency of each type is expressed in percentage and the number of clones obtained for each type is indicated in parenthesis.

Effect of IEE on other types of IS elements. Using a reporter plasmid-based assay systems similar to that shown in Figure 1a, we examined whether IEE promotes the excision of other types of IS elements: two other IS3 family members (IS2 and IS3) and six IS elements each belonging to different families (IS1, IS4, IS5, IS26, IS30 and IS621; Fig. 6). The excision of IS2 and IS3 was promoted by IEE to the same level as that for IS629, suggesting that the IS excisionenhancing activity of IEE may cover the entire IS3 family. Among the other IS elements, the excision frequencies of IS1 and IS30 also clearly increased in the presence of IEE, although to a lesser extent compared with that of the IS3 family. Thus, IEE enhances the excision of (minimally) members of the IS3, IS1 and IS30 a

First strand cleavage b

Strand transfer

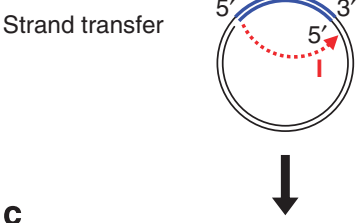

Figure-eight formation

d

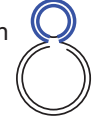

Resolution of the figure eight

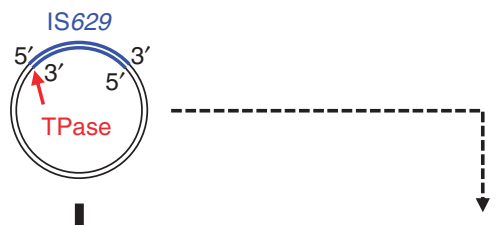

e

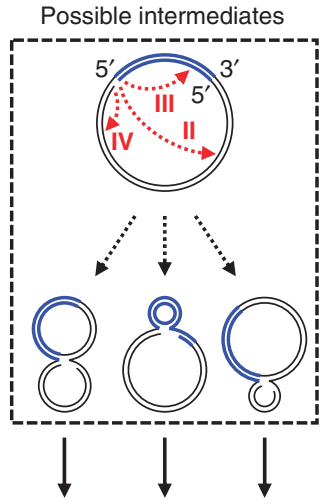

Second strand cleavage

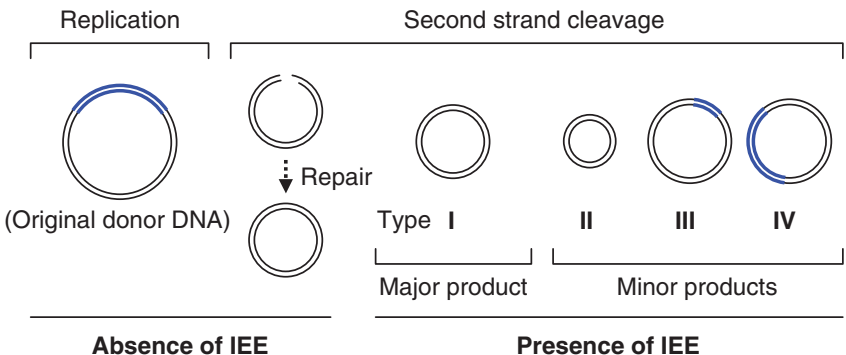

Figure 4 | Possible pathways for IS629 excision and for the generation of various types of genomic deletions on IS excision. Although the process of IS629 transposition has not been characterized, it is most likely that it transposes in a process similar to that of other IS3 family members ${ }^{7}$. (a) TPase first cleaves one strand at the $3^{\prime}$ end of IS629 (thick blue line) as indicated by the red arrow. (b) The liberated $3^{\prime}$ end is transferred to the proximity of the $5^{\prime}$ end of IS629 (usually 3 or 4 bp away from the $5^{\prime}$ end) on the same strand, as indicated by the dotted red arrow I. (c) The strand transfer event generates a figure-eight structure in which the ends of IS629 are joined by a single-stranded bridge. (d) DNA replication from the figure-eight junction generates a circular transposition intermediate and regenerates the donor DNA containing the IS copy. It is unknown whether liberation of the circle by some other mechanism(s), such as second-strand cleavage, occurs in the normal transposition process. If this does happen, the ends of the linearized donor DNA would need to be repaired for its survival. In the presence of IEE, various types of genomic deletions occur at IS insertion sites. The major product is a type I deletion representing simple excision, and other types of genomic deletions (types II, III and IV) are minor products. (e) If aberrant strand transfer (to a genome position far away from the $5^{\prime}$ end of IS629, inside of IS629, or very far from the $5^{\prime}$ end (that is, near the $3^{\prime}$ end of IS629, where the first strand cleavage occurred)) occurred, as indicated by dotted arrows II, III and IV, they would be intermediates for the formation of each variant type of genomic deletion.

families. Although it is unknown whether a figure-eight intermediate is formed during IS1 transposition, IS30 has been shown to form this structure ${ }^{25}$. This raises the possibility that IEE may function on a wide range of IS elements that transpose through the formation of figure-eight intermediates.

It is noteworthy that the effect of IEE on IS1 was significantly less pronounced than that on IS3 family members and IS30. This phenomenon may be related to the finding that IS1 appears to be able to use two pathways for transposition ${ }^{26}$ : one involves excision 
of a circular transposition intermediate (similar to the IS3 family) and thus would be expected to be IEE sensitive, whereas the other involves cointegrate formation and would not be sensitive to IEE.

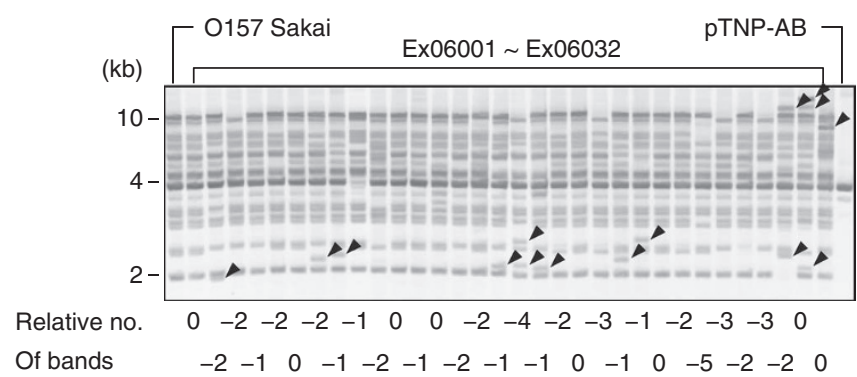

Figure 5 | Southern hybridization analysis of 0157 Sakai and clones Ex06001-Ex06032 using an IS629-specific probe. To determine the difference in the total number of IS629 copies in clones Ex06001Ex06032, Southern hybridization analysis using an IS629-specific probe was performed. Sspl-digested genomic DNA was separated by pulsed-field gel electrophoresis and subjected to Southern hybridization analysis. The DNA probe used was derived from the central part (positions 308-607) of IS629. The differences in the total copy number deduced for each clone (relative to that of the parent strain 0157 Sakai) are indicated at the bottom. Signals that are detected in each clone but not in the parent strain are indicated by arrows. Only pTNP-AB plasmid DNA was applied to the lane labelled pTNP-AB. Note that the total copy number did not increase in any of the 32 clones.

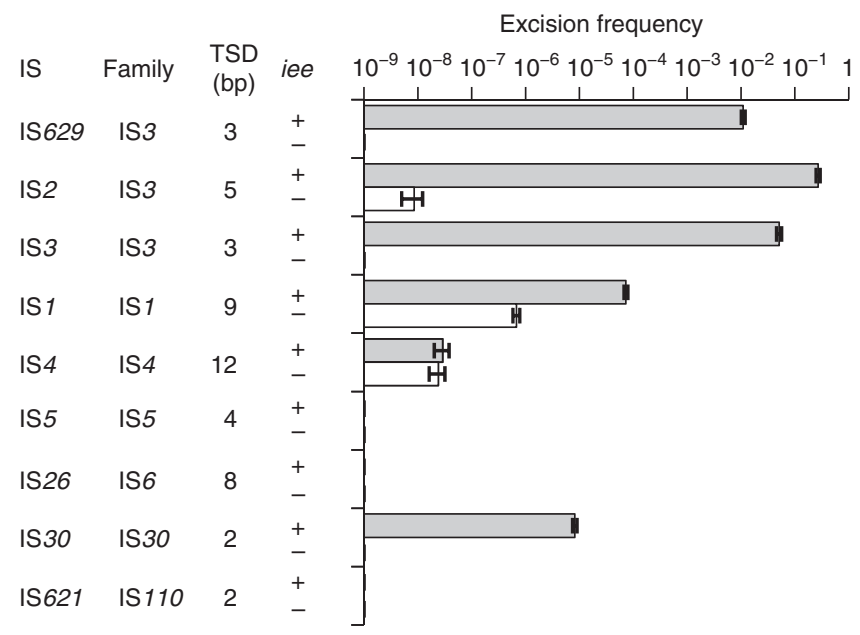

Figure 6 | Excision frequencies of various IS elements in the presence or absence of IEE. Excision frequencies of IS1, IS2, IS3, IS4, IS5, IS26, IS 30 and IS621 in the presence or absence of IEE were examined in K-12 using reporter plasmids similar to $\mathrm{pDN}-\mathrm{TcR}$ but comprising a cognate TPase coding region and TIR regions. The excision frequency was determined by dividing the number of ampicillin-resistant $\left(A p^{r}\right)$ colonies by tetracyclineresistant $\left(T^{r}\right.$ ) colonies (mean \pm s.d., $n=3$ ). The IS family to which each IS belongs and the lengths of target site duplication (TSD) generated by each IS are indicated according to the descriptions in ISfinder ${ }^{5}$ (http://Wwwis.biotoul.fr/). Plus and minus symbols indicate the presence and absence, respectively, of the iee gene on the additional plasmid. The difference of the excision frequencies of IS4 (pIEE1 +) and IS4 (pIEE1 -) was not statistically significant $(P<0.01)$. No Apr colony was obtained for IS629 (pIEE1-), IS3 (pIEE1-), IS5 (pIEE1+/-), IS26 (pIEE1+/-), IS30 (pIEE1-) or IS621 $($ pIEE $1+/-)$.
Wide distribution of IEE homologues and their phylogenies. The iee gene is located in a large integrative element, SpLE1, in O157 EHEC and in SpLE1-like elements in O26, O111 and O103 EHECs. These mobile genetic elements are specifically distributed in these EHEC strains ${ }^{27}$ (Supplementary Fig. S5), and the iee gene is not present in other sequenced $E$. coli strains, with the single exception of strain EDla (ref. 28). Thus, IEE shows a strain-specific distribution in E. coli. In ED-1, a commensal E. coli strain, the iee gene is located in a different type of genetic element that has been integrated into a tRNA gene (thrW) (Supplementary Fig. S5).

A BLASTP search of the GenBank NR database with filtering of results by hit-length coverage $(>80 \%)$ and sequence identity ( $>30 \%$ ) identified many IEE homologues from a broad range of bacteria. Phylogenetic analysis of these IEE homologues revealed that the homologues from different phyla often cluster together (Figure 7; different phyla are indicated by circles of different colour). This finding indicates that the evolution of IEE homologues did not follow that of the host genomes. Furthermore, the distribution of IEE homologues suggests that they have spread in each species in a strain-specific manner, as observed in E. coli. For example, among the four fully sequenced Legionella pneumophila strains, IEE homologues were found in only two. In Bacteroides fragilis, Desulfitobacterium hafniense, Lactobacillus rhamnosus and Streptococcus pneumoniae as well, IEE homologues were found only in one strain among the two or more sequenced strains. In these five species, genes for their IEE homologues were located in strain-specific regions or genomic islands (Supplementary Fig. S6).

These results suggest that IEE homologues have been spread to a variety of bacteria strains by horizontal gene transfer. To gain further insight into this issue, we analysed the genomic locations of all the other 24 IEE homologues that were identified in bacteria species, of which only a single strain has so far been fully sequenced (Supplementary Fig. S7). Among the homologues analysed, many (at least 19 of 24) were encoded in genomic regions exhibiting low GC content and/or containing genes related to mobile elements, such as phage, Tn or IS. Furthermore, two additional IEE homologues were found on plasmids (Dinoroseobacter shibae strain DFL12 and Caulobacter sp. strain K31). These results support the notion that IEE homologues have been spread widely in bacteria by horizontal gene transfer.

The identification of many IEE homologues allowed us to perform multiple sequence alignment analysis (Supplementary Fig. S8). From this analysis, we found four regions that are highly conserved between the IEE family of proteins (regions 1-4). A domain search using SMART ${ }^{29}$ and Pfam $^{30}$ revealed that regions 1 and 2 exhibit some similarity to a sequence motif found in eukaryotic/archaeal primases (pfam01896), whereas regions 3 and 4 contain a DEXDc motif (pfam00270) and a HELICc motif (smart00490), respectively, which are found in protein families such as the DEAD and DEAH box helicases. Although the relationship between IEE function and these motifs must be elucidated in future, our preliminary results indicate that alanine substitution of several invariant amino-acid residues found in regions 3 and 4 considerably reduces IEE activity to promote IS629 excision.

\section{Discussion}

In this study, we showed that IEE promotes the excision of IS elements belonging to the IS3 family, as well as those belonging to the IS1 and IS30 families, from bacterial genomes. Although the molecular mechanism of IEE action remains unknown, the identification of IEE is scientifically important from several perspectives. First, IEE is the first bacterial protein that has been shown to promote IS excision in a TPase or transposition-dependent manner; although it may not function directly on TPase, it may function on an intermediate generated during transposition. 


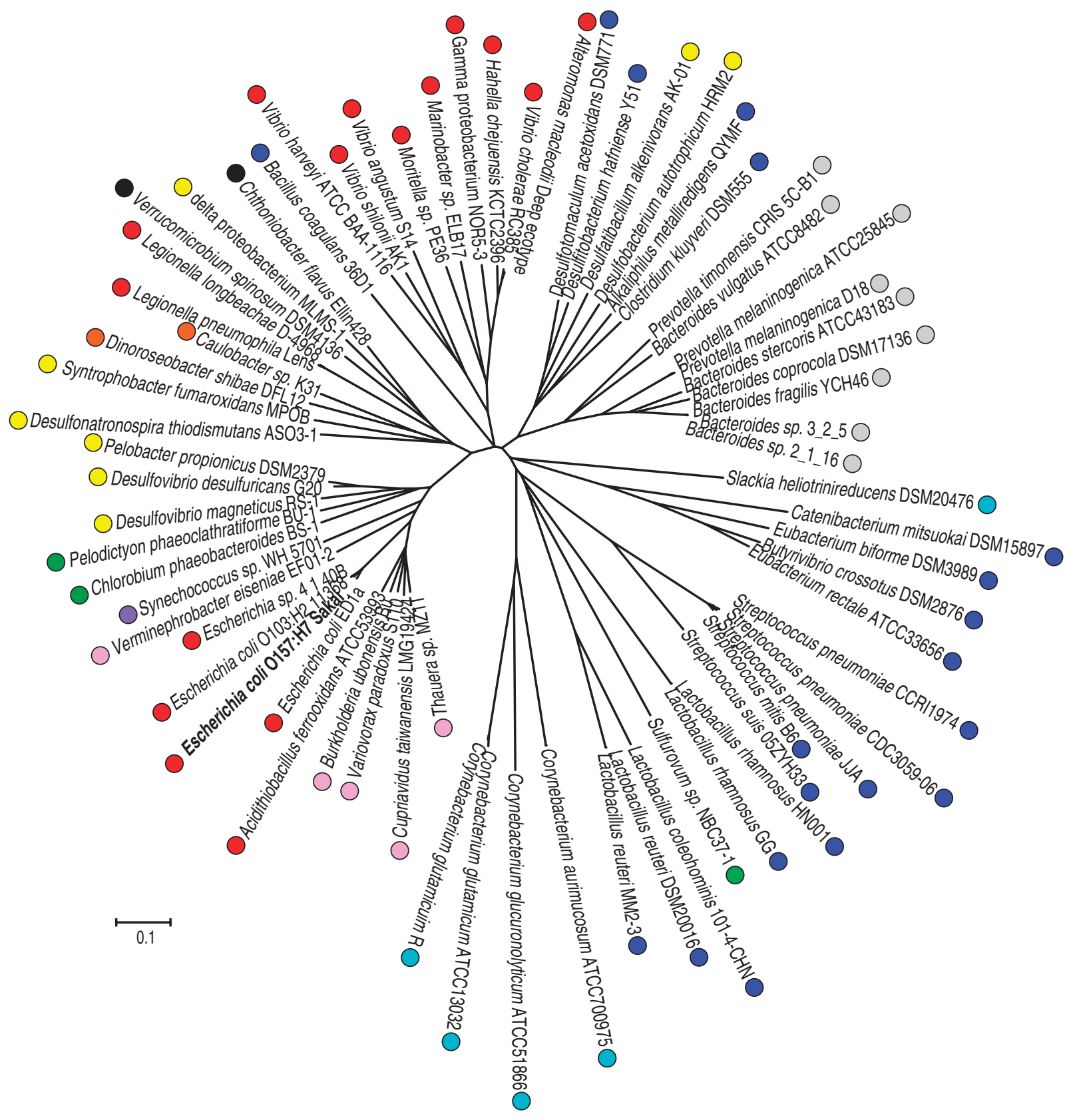

Figure 7 | Phylogeny of IEE homologues. IEE homologues were identified by a BLASTP search of the GenBank NR database with filtering of the results by hit-length coverage ( $>80 \%$ ) and sequence identity ( $>30 \%$ ). Amino-acid sequences of the IEE homologues were aligned using CLUSTALW in MEGA4 software ${ }^{49}$, and a neighbour-joining tree was generated with a bootstrap of 1,000 replicates. The names of the bacterial species and strains in which each homologue was discovered are shown. The phylum (or class) of the species is indicated by coloured circles: orange, Alphaproteobacteria; pink, Betaproteobacteria; red, Gammaproteobacteria; yellow, Deltaproteobacteria; light green, Epsilonproteobacteria; grey, Bacteroides; green, Chlorobi; violet, Cyanobacteria; blue, Firmicutes; light blue, Actinobacteria; and black, Verrucomicrobia. The size bar represents the number of substitutions per site. The IEE homologues from different phyla often cluster together, indicating that the evolution of the IEE homologues did not follow that of their host genomes.

Several host factors, such as proteins for DNA repair and recombination, may also induce the deletion of $\mathrm{Tns}^{31}$ but in a TPase-independent manner and at much lower efficiencies compared with that promoted by IEE (Supplementary Fig. S2). This function of IEE is particularly important for bacterial cells because, unlike eukaryotic cells, most bacterial species have no specific end-joining activity to regenerate and protect the donor genome after IS excision. Second, IEE-mediated IS excision generates a variety of genomic deletions. IS elements and their transpositions are generally regarded as one of the major driving forces generating various genomic deletions, but precise mechanisms remain unknown for many of the genomic deletions detected in sequenced bacterial genomes. The action of IEE can explain how genomic deletions are generated on IS transposition or excision. Third, structural features of the donor DNA regenerated after IEE-induced IS excision, particularly for variant types of deletions, may provide further insight into the process of IS transposition. Processes leading to the generation of such variant types of genomic deletions may take place only when IEE is present. However, it is also possible that rare or aberrant intermediates that are unable to survive in the normal process of transposition (that is, in the absence of IEE) may be trapped by the action of IEE. If so, the structures of these IEE-induced deletions may represent 
or reflect the structures of rare intermediates generated in the normal transposition process. Finally, IEE can be regarded as a new type of genetic system that controls the copy number of Tns in host genomes.

The invasion and proliferation of Tns are important in long-term genome evolution, but their uncontrolled proliferation is sometimes detrimental to the host. Thus, various genetic systems to suppress or attenuate their transposition activities have evolved. Tc1 transposition is silenced by RNA interference in the Caenorhabditis elegans germ line ${ }^{32}$. This system also suppresses the transposition of other types of Tns, such as Tc3, Tc5 and Tc7 (ref. 33). Silencing of Tns is also achieved by epigenetic mechanisms through modification of histone tails, DNA methylation and chromatin remodelling ${ }^{34}$. In addition, the transposition activities of some Tns are negatively regulated by a repressor encoded by Tns themselves, as seen in the $P$ element of Drosophila ${ }^{35}$ and in several bacterial Tns, such as Tn5 (ref. 36), IS1 (refs 37, 38) and members of the IS3 family ${ }^{39}$. The coexpression of IEE and of IS629 TPase reduced the copy number of IS629 in O157, and this could happen to other members of the IS3 family and to members of (minimally) the IS1 and IS30 families. This type of control mechanism has not been described in eukaryotes, archaea or bacteria.

The identification of many IEE homologues from a broad range of bacterial species represents an important finding in terms of gene evolution. The results of the phylogenetic analysis of these IEE homologues indicate that their evolution did not follow that of their host genomes, instead suggesting the involvement of horizontal gene transfer in the spread of IEE homologues among bacteria. This idea is supported by the observation that IEE homologues from several bacterial species for which multiple genome sequences are available clearly show a strain-specific distribution, and many of the other IEE homologues are also encoded in genomic regions of apparently foreign origin. Thus, IEEs may represent a novel protein family that has been widely spread by horizontal gene transfer and has coevolved with a number of IS elements belonging to the IS3 and other families. Further, these proteins may have pivotal roles in the evolution of many bacterial species or strains by inducing IS excision and various types of genomic deletions.

\section{Methods}

Bacterial strains and culture conditions. RIMD0509952 (referred to as O157 Sakai) is an EHEC O157:H7 strain isolated from a typical patient during the Sakai outbreak that occurred in Japan in 1996. MG1655 (referred to as K-12) is an E. coli $\mathrm{K}-12$ strain maintained as a laboratory strain with minimal genetic manipulation. The genome sequences of both $\mathrm{O} 157$ Sakai and K-12 were previously determined $^{19,40}$. The other EHEC O157, O26, O111 and O103 strains used for the analysis of excision frequencies (Fig. 1b) were also clinical isolates ${ }^{41,42}$. GMSS401 is an isogenic mutant of O157 Sakai in which ECs1305 was deleted from the chromo some. GMEC101 is an isogenic mutant of K-12 in which ECs1305 was inserted in an IS3 copy on the chromosome. The isogenic mutants were generated using the method developed by Djafari et al. ${ }^{43}$

All strains were grown in LB broth ${ }^{44}$ supplemented, when necessary, with $50 \mu \mathrm{g} \mathrm{ml}^{-1}$ ampicillin (Ap), $30 \mu \mathrm{g} \mathrm{ml}^{-1}$ chloramphenicol (Cm), $20 \mu \mathrm{g} \mathrm{ml}^{-1} \mathrm{kana}^{-}$ mycin $(\mathrm{Km})$ or $20 \mu \mathrm{g} \mathrm{ml}^{-1}$ tetracycline $(\mathrm{Tc})$. The selection of mutants was carried out on LB agar plates supplemented with the appropriate antibiotic(s).

DNA manipulations. Restriction endonucleases and T4 DNA ligase were purchased from Takara Bio. E. coli cells were transformed by electroporation ${ }^{44}$. Plasmid DNA was prepared using a QIAfilter Plasmid kit (Qiagen). Nucleotide sequences were determined by the dideoxy chain termination method $^{45}$ using a BigDye Terminator Cycle Sequencing kit and an Applied Biosystems 3130 sequencer (Applied Biosystems) according to the manufacturer's instructions.

Construction of plasmids. pDN-TcR was used as a reporter plasmid in the IS629 excision assay (Fig. 1a). In this plasmid, IS629-Tc and the IS629 TPase gene are inserted in the $\mathrm{Ap}^{\mathrm{r}}$ gene ( $\mathrm{ScaI}$ site) and the HincII site of pUC18, respectively ${ }^{18}$. IS629-Tc is an IS629 analogue, in which the IS629 TPase gene was replaced with the $\mathrm{Tc}^{\mathrm{r}}$ gene from pBR322 (ref. 18). pTNP-AB is an IS629 TPase expression plasmid carrying a part of IS629 comprising the -10 and -35 promoter region, the Shine-Dalgarno sequence and the TPase coding region ${ }^{18}$.

The reporter plasmids for analysing other types of IS elements shown in Figure 6 were constructed by substituting the TPase coding region and the TIR regions of IS629 in pDN-TcR with those of each cognate IS element. The TPase coding regions and the TIR regions of other IS elements were amplified by PCR using the primers listed in Supplementary Table S1. The amplicons were then used as pairs of complementary primers in the PCR-based site-directed mutagenesis method $^{46}$. PCR was performed in a $50 \mu \mathrm{l}$ reaction mixture containing $50 \mathrm{ng}$ of template DNA (pDN-TcR), $100 \mathrm{ng}$ of amplicon, $0.2 \mathrm{mM}$ of each deoxynucleoside triphosphate, PCR buffer and $1 \mathrm{U}$ of KOD-plus DNA polymerase (Toyobo) with 18 cycles of $15 \mathrm{~s}$ at $94^{\circ} \mathrm{C}, 30 \mathrm{~s}$ at $60^{\circ} \mathrm{C}$ and $5.5 \mathrm{~min}$ at $68^{\circ} \mathrm{C}$. IS 1 , IS2 and IS3 have two consecutive and partially overlapping open reading frames (ORFs), orf $A$ and orf $B$ (ins $A$ and ins $B$ for IS 1 ), positioned in the 0 and -1 phases, respectively. Because, as in IS629 (ref. 17), the TPases of these IS elements are produced as a fused protein OrfAB (or InsAB'), by programmed frameshifting between the two ORFs ${ }^{2}$, a 1-bp insertion was introduced in the overlapping region of the two ORFs of IS1, IS2 and IS3 by PCR-based site-directed mutagenesis ${ }^{46}$ using pairs of complementary primers IS1-SF and IS1-SR, IS2-SF and IS2-SR, and IS3-SF and IS3-SR, respectively (Supplementary Table S1).

pBAD-IEE is a plasmid in which ECs1305 (the iee gene) was inserted into the SmaI site of pBAD33 (ref. 47), and expression of the iee gene is induced by L-arabinose. The coding region of ECs 1305 was amplified by PCR with two primers (5'-AGGAGGAATTCACCATGGTTCATAAATCTGACAGTGATGAATTAG-3' and $5^{\prime}$-TCATTAAATCACTGATTCATCGCCGTCAGC- $3^{\prime}$ ) and used for plasmid construction. PCR was performed in a $50 \mu \mathrm{l}$ reaction mixture containing template DNA (genomic DNA of O157 Sakai), $0.3 \mu \mathrm{M}$ each of the two primers, $0.2 \mathrm{mM}$ of each deoxynucleoside triphosphate, PCR buffer and $1 \mathrm{U}$ of KOD-plus DNA polymerase (Toyobo) with 25 cycles of $15 \mathrm{~s}$ at $94^{\circ} \mathrm{C}, 30 \mathrm{~s}$ at $60^{\circ} \mathrm{C}$ and $25 \mathrm{~min}$ at $68^{\circ} \mathrm{C}$. By nucleotide sequencing, we confirmed that no unexpected mutation was introduced into the cloned ECs1305 fragment. pBAD33 was provided by the National BioResource Project (NIG, Japan): E. coli.

Analysis of structural alterations in IS629 insertion sites. O157 Sakai(pTNP$\mathrm{AB} / \mathrm{pBAD}$-IEE), which is an O157 Sakai derivative containing both pTNP-AB and pBAD-IEE, was cultivated at $37^{\circ} \mathrm{C}$ for $24 \mathrm{~h}$ in LB broth containing $0.2 \% \mathrm{~L}$-arabinose to induce iee gene expression. The bacterial cells were then grown on an $\mathrm{LB}$ plate, and 32 single colonies were randomly selected (Ex06001-Ex06032). Structural alterations in all but two IS629 insertion sites in the genomes of these 32 clones were analysed using the IS-printing kit (Toyobo) according to the manufacturer's instructions. The two IS629 insertion sites were analysed using primers $5^{\prime}$-CAGT GGATGCCAATAAGCCAG-3' and 5'-GAGACACAATGCCCATCCTTCG-3', respectively. Similar analyses were performed for another three sets of O157 Sakaiderived clones (Ex07001-Ex07032, Ex08001-Ex08032 and Ex09001-Ex09032), which were derived from O157 Sakai(pTNP-AB/pBAD33), O157 Sakai(pHSG298/ pBAD-IEE) and O157 Sakai(pHSG298/pBAD33), respectively. pHSG298 and pBAD33 were used as controls for pTNP-AB and pBAD-IEE, respectively.

To determine the altered structures of each IS629 insertion site, we amplified the genomic segments containing the IS629 insertion site from Ex06001 to Ex06032 by PCR using primer sets for whole-genome PCR scanning (a long-range PCR-based genome comparison system developed by Ohnishi et al. ${ }^{41}$ ), and determined the nucleotide sequences of IS629-flanking regions.

PCR analysis of IS elements other than IS629. All O157 Sakai genomic loci with insertion of intact IS elements other than IS629 (non-IS629) were examined by PCR using primers shown in Supplementary Table S2. PCR was performed in a $50 \mu \mathrm{l}$ reaction mixture containing template DNA (genomic DNA of O157 Sakai or clones Ex06001-Ex06032), 0.2 $\mu \mathrm{M}$ of each primer, $0.2 \mathrm{mM}$ of each deoxynucleoside triphosphate, PCR buffer and $1.25 \mathrm{U}$ of Ex Taq DNA polymerase (Takara Bio) with 35 amplification cycles of $30 \mathrm{~s}$ at $95^{\circ} \mathrm{C}, 30 \mathrm{~s}$ at $60^{\circ} \mathrm{C}$ and $4 \mathrm{~min}$ at $72^{\circ} \mathrm{C}$.

Pulsed-field gel electrophoresis. Pulsed-field gel electrophoresis was performed by clamped homogeneous electric field electrophoresis using a CHEFF DR III apparatus (Bio-Rad Laboratories). Genomic DNA of each strain was prepared by the method described by Akiba et al..$^{48}$ Genomic DNA in sliced plugs was digested with $30 \mathrm{U}$ of $S s p \mathrm{I}$ at $37^{\circ} \mathrm{C}$ for $16 \mathrm{~h}$. Electrophoresis was conducted in a $1 \%$ agarose gel in $0.5 \times$ Tris-borate-EDTA buffer at $14^{\circ} \mathrm{C}$ at $6 \mathrm{~V} \mathrm{~cm}^{-1}$ for $10 \mathrm{~h}$ with a pulse time of $4 \mathrm{~s}$.

Southern blot hybridization analysis. A digoxigenin (DIG)-labelled PCR product was used as a DNA probe. A 300-bp fragment covering the central part of IS629 was amplified by PCR in a $100-\mu 1$ reaction mixture containing template DNA (genomic DNA of the O157 Sakai strain), $0.2 \mu \mathrm{M}$ each of two primers (5'-GCAG TAACGATATCCTTCGCCAGGC-3' and 5'-CGTAACAACTGACGCCAGACTT TACGC-3'), $10 \mu$ l of PCR DIG Labeling Mix (Roche Diagnostics), PCR buffer and $1.25 \mathrm{U}$ of Ex Taq DNA polymerase (Takara Bio) for 30 amplification cycles of $94^{\circ} \mathrm{C}$ for $30 \mathrm{~s}, 60^{\circ} \mathrm{C}$ for $30 \mathrm{~s}$ and $72^{\circ} \mathrm{C}$ for $30 \mathrm{~s}$. The electrophoresed DNA was blotted onto a Hybond-N + charged nylon membrane (GE Healthcare) by the standard method ${ }^{44}$. The blotted membrane was then probed with the DIG-labelled PCR product. Prehybridization and hybridization were carried out at $68^{\circ} \mathrm{C}$ using PerfectHyb hybridization solution (Toyobo), and after washes with buffer A ( $2 \times$ SSC with $0.1 \%$ SDS $)$ and buffer B $(0.1 \times$ SSC with $0.1 \%$ SDS $)$, signals were detected using a DIG Nucleic Acid Detection kit (Roche Diagnostics) according to the manufacturer's instructions. 


\section{References}

1. Sinzelle, L., Izsvak, Z. \& Ivics, Z. Molecular domestication of transposable elements: from detrimental parasites to useful host genes. Cell Mol. Life Sci. 66, 1073-1093 (2009)

2. Chandler, M. \& Mahillon, J. Mobile DNA II (eds Craig, N. L., Craigie, R., Gellert, M. \& Lambowitz, A. M.) 305-366 (ASM Press, 2002).

3. Wei, J. et al. Complete genome sequence and comparative genomics of Shigella flexneri serotype 2a strain 2457T. Infect. Immun. 71, 2775-2786 (2003).

4. Kothapalli, S. et al. Diversity of genome structure in Salmonella enterica serovar Typhi populations. J. Bacteriol. 187, 2638-2650 (2005).

5. Siguier, P., Perochon, J., Lestrade, L., Mahillon, J. \& Chandler, M. ISfinder: the reference centre for bacterial insertion sequences. Nucleic Acids Res. 34, D32-36 (2006).

6. Mahillon, J. \& Chandler, M. Insertion sequences. Microbiol. Mol. Biol. Rev. 62, 725-774 (1998).

7. Rousseau, P. et al. Mobile DNA II (eds Craig, N. L., Craigie, R., Gellert, M. \& Lambowitz, A. M.) 367-383 (ASM Press, 2002).

8. Synder, L. \& Champness, W. Molecular Genetics of Bacteria (ASM Press, 2003).

9. Stephanou, N. C. et al. Mycobacterial nonhomologous end joining mediates mutagenic repair of chromosomal double-strand DNA breaks. J. Bacteriol. 189, 5237-5246 (2007)

10. Foster, T. J., Lundblad, V., Hanley-Way, S., Halling, S. M. \& Kleckner, N. Three Tn10-associated excision events: relationship to transposition and role of direct and inverted repeats. Cell 23, 215-227 (1981).

11. Nagel, R. \& Chan, A. Enhanced Tn 10 and mini-Tn 10 precise excision in DNA replication mutants of Escherichia coli K12. Mutat. Res. 459, 275-284 (2000).

12. Mead, P. S. \& Griffin, P. M. Escherichia coli O157:H7. Lancet 352, 1207-1212 (1998).

13. Makino, K. et al. Complete nucleotide sequences of $93-\mathrm{kb}$ and $3.3-\mathrm{kb}$ plasmids of an enterohemorrhagic Escherichia coli O157:H7 derived from Sakai outbreak. DNA Res. 5, 1-9 (1998).

14. Ooka, T. et al. Inference of the impact of insertion sequence (IS) elements on bacterial genome diversification through analysis of small-size structural polymorphisms in Escherichia coli $\mathrm{O} 157$ genomes. Genome Res. 19, 1809-1816 (2009).

15. Kusumoto, M., Nishiya, Y., Kawamura, Y. \& Shinagawa, K. Identification of an insertion sequence, IS1203 variant, in a Shiga toxin 2 gene of Escherichia coli O157:H7. J. Biosci. Bioeng. 87, 93-96 (1999).

16. Okitsu, T. et al. Identification of shiga toxin-producing Escherichia coli possessing insertionally inactivated Shiga toxin gene. Microbiol. Immunol. 45, 319-322 (2001).

17. Kusumoto, M., Nishiya, Y. \& Kawamura, Y. Reactivation of insertionally inactivated Shiga toxin 2 genes of Escherichia coli O157:H7 caused by nonreplicative transposition of the insertion sequence. Appl. Environ. Microbiol. 66, 1133-1138 (2000)

18. Kusumoto, M., Suzuki, R., Nishiya, Y., Okitsu, T. \& Oka, M. Host-dependent activation of IS1203v excision in Shiga toxin-producing Escherichia coli. J. Biosci. Bioeng. 97, 406-411 (2004).

19. Hayashi, T. et al. Complete genome sequence of enterohemorrhagic Escherichia coli O157:H7 and genomic comparison with a laboratory strain K-12. DNA Res. 8, 11-22 (2001).

20. Ogura, Y. et al. Complexity of the genomic diversity in enterohemorrhagic Escherichia coli $\mathrm{O} 157$ revealed by the combinational use of the O157 Sakai OligoDNA microarray and the Whole Genome PCR scanning. DNA Res. 13, 3-14 (2006)

21. Nagy, Z. \& Chandler, M. Regulation of transposition in bacteria. Res. Microbiol. 155, 387-398 (2004).

22. Ooka, T. et al. Development of a multiplex PCR-based rapid typing method for enterohemorrhagic Escherichia coli O157 strains. J. Clin. Microbiol. 47, 2888-2894 (2009)

23. Sekine, Y., Aihara, K. \& Ohtsubo, E. Linearization and transposition of circular molecules of insertion sequence IS3. J. Mol. Biol. 294, 21-34 (1999).

24. Duval-Valentin, G., Marty-Cointin, B. \& Chandler, M. Requirement of IS911 replication before integration defines a new bacterial transposition pathway. EMBO J. 23, 3897-3906 (2004).

25. Szabo, M., Kiss, J., Nagy, Z., Chandler, M. \& Olasz, F. Sub-terminal sequences modulating IS30 transposition in vivo and in vitro. J. Mol. Biol. 375, 337-352 (2008)

26. Turlan, C. \& Chandler, M. IS1-mediated intramolecular rearrangements: formation of excised transposon circles and replicative deletions. EMBO J. 14, 5410-5421 (1995)

27. Ogura, Y. et al. Comparative genomics reveal the mechanism of the parallel evolution of $\mathrm{O} 157$ and non-O157 enterohemorrhagic Escherichia coli. Proc. Natl Acad. Sci. USA 106, 17939-17944 (2009).

28. Touchon, M. et al. Organised genome dynamics in the Escherichia coli species results in highly diverse adaptive paths. PLoS Genet. 5, e1000344 (2009).

29. Schultz, J., Milpetz, F., Bork, P. \& Ponting, C. P. SMART, a simple modular architecture research tool: identification of signaling domains. Proc. Natl Acad. Sci. USA 95, 5857-5864 (1998).

30. Finn, R. D. et al. The Pfam protein families database. Nucleic Acids Res. 36, D281-288 (2008).
31. Lundblad, V., Taylor, A. F., Smith, G. R. \& Kleckner, N. Unusual alleles of $r e c B$ and $\mathrm{recC}$ stimulate excision of inverted repeat transposons $\operatorname{Tn} 10$ and $\mathrm{Tn} 5$. Proc. Natl Acad. Sci. USA 81, 824-828 (1984).

32. Sijen, T. \& Plasterk, R. H. Transposon silencing in the Caenorhabditis elegans germ line by natural RNAi. Nature 426, 310-314 (2003).

33. Ketting, R. F., Haverkamp, T. H., van Luenen, H. G. \& Plasterk, R. H. mut-7 of C. elegans, required for transposon silencing and RNA interference, is a homolog of Werner syndrome helicase and RNaseD. Cell 99, 133-141 (1999).

34. Slotkin, R. K. \& Martienssen, R. Transposable elements and the epigenetic regulation of the genome. Nat. Rev. Genet. 8, 272-285 (2007).

35. Rio, D. C., Laski, F. A. \& Rubin, G. M. Identification and immunochemical analysis of biologically active Drosophila P element transposase. Cell 44, 21-32 (1986).

36. Johnson, R. C., Yin, J. C. \& Reznikoff, W. S. Control of Tn 5 transposition in Escherichia coli is mediated by protein from the right repeat. Cell 30, 873-882 (1982).

37. Machida, C. \& Machida, Y. Regulation of IS 1 transposition by the ins A gene product. J. Mol. Biol. 208, 567-574 (1989)

38. Zerbib, D., Polard, P., Escoubas, J. M., Galas, D. \& Chandler, M. The regulatory role of the IS1-encoded InsA protein in transposition. Mol. Microbiol. 4, 471-477 (1990).

39. Sekine, Y., Izumi, K., Mizuno, T. \& Ohtsubo, E. Inhibition of transpositional recombination by OrfA and OrfB proteins encoded by insertion sequence IS3. Genes Cells 2, 547-557 (1997)

40. Blattner, F. R. et al. The complete genome sequence of Escherichia coli K-12. Science 277, 1453-1474 (1997).

41. Ohnishi, M. et al. Genomic diversity of enterohemorrhagic Escherichia coli O157 revealed by whole genome PCR scanning. Proc. Natl Acad. Sci. USA 99, 17043-17048 (2002).

42. Ogura, Y. et al. Extensive genomic diversity and selective conservation of virulence-determinants in enterohemorrhagic Escherichia coli strains of O157 and non-O157 serotypes. Genome Biol. 8, R138 (2007).

43. Djafari, S., Hauf, N. D. \& Tyczka, J. F. Generation of isogenic deletion mutants of STEC. Methods Mol. Med. 73, 113-124 (2003).

44. Sambrook, J. \& Russel, D. W. Molecular Cloning (Cold Spring Harbor Laboratory, 2001).

45. Sanger, F., Nicklen, S. \& Coulson, A. R. DNA sequencing with chainterminating inhibitors. Proc. Natl Acad. Sci. USA 74, 5463-5467 (1977).

46. Zheng, L., Baumann, U. \& Reymond, J. L. An efficient one-step site-directed and site-saturation mutagenesis protocol. Nucleic Acids Res. 32, e115 (2004).

47. Guzman, L. M., Belin, D., Carson, M. J. \& Beckwith, J. Tight regulation, modulation, and high-level expression by vectors containing the arabinose $\mathrm{P}_{\mathrm{BAD}}$ promoter. J. Bacteriol. 177, 4121-4130 (1995)

48. Akiba, M. et al. Comparison of Salmonella enterica serovar Abortusequi isolates of equine origin by pulsed-field gel electrophoresis and fluorescent amplifiedfragment length polymorphism fingerprinting. Vet. Microbiol. 92, 379-388 (2003).

49. Tamura, K., Dudley, J., Nei, M. \& Kumar, S. MEGA4: molecular evolutionary genetics analysis (MEGA) software version 4.0. Mol. Biol. Evol. 24, 1596-1599 (2007).

\section{Acknowledgments}

This work was supported by a Grant-in-Aid for Scientific Research on Priority Areas 'Applied Genomics' from the Ministry of Education, Science and Technology of Japan. We thank H. Ohtani for technical assistance.

\section{Author contributions}

M.K. and T.H. conceived and designed the study, analysed the results and wrote the paper. M.K. carried out most of the experiments, with contributions from Y.N., T.S., Y.S., T.I. and M.A. T.O. obtained the results shown in Figure 7 and in Supplementary Figures S3, S5-S8. Y.O. analysed the comparative genomic hybridization data. All authors discussed and commented on the paper.

\section{Additional information}

Data deposition: The nucleotide sequence of pBAD-IEE has been deposited in DDBJ/EMBL/GenBank under accession number AB598835.

Supplementary Information accompanies this paper at http://www.nature.com/ naturecommunications

Competing financial interests: The authors declare no competing financial interests.

Reprints and permission information is available online at http://npg.nature.com/ reprintsandpermissions/

How to cite this article: Kusumoto, M. et al. Insertion sequence-excision enhancer removes transposable elements from bacterial genomes and induces various genomic deletions. Nat. Commun. 2:152 doi: 10.1038/ncomms1152 (2011). 